\title{
Amending Federal Regulations to Counteract Language Barriers in the Informed Consent Process
}

\author{
Suzanne Mistretta
}

ABSTRACT

As English is the predominant language of research protocols in the United States, non-English speaking subjects face language barriers during clinical trial enrollment. Federal regulation 45 C.F.R. 46 requires that a research subject receive information about a clinical trial "in language understandable to the subject or the legally authorized representative." A researcher may enroll a subject using short-form consent when a longform translation in the subject's native language is not available. However, the abbreviated short form does not adequately inform the subject of the study's purpose and potential risks. United States Department of Health and Human Services (HHS) leaders should amend federal guidance to provide specific details on obtaining proper informed consent when there is a language barrier. The code of federal regulations should also establish a standard for quality translation services and interpreters. This paper will review current federal regulations and draft policy, analyze literature describing hospital experiences, and discuss noncompliance areas. This author recommends an amendment to federal policy, which is important because it helps ensure the rights of study participants under the principle of justice.

Keywords: Research Ethics, Language Barriers, Clinical Trials, Informed Consent

\section{INTRODUCTION}

As English is the predominant language of research protocols in the United States, non-English speaking subjects face language barriers during clinical trial enrollment. Law requires that a research subject should receive information about a clinical trial "in language understandable to the subject or the legally authorized representative." ${ }^{1}$ This law states that a researcher may enroll a subject using a "short-form" consent when a "long-form" translation in the subject's native language is not available. ${ }^{2}$ However, the abbreviated short form does not adequately inform the subject of the study's purpose and potential risks. Furthermore, the law does not outline a standard for quality translators. United States Department of Health and Human Services (HHS) leaders should amend federal guidance to provide specific details on obtaining proper informed consent when there is a language barrier. ${ }^{3}$ The Code of Federal Regulations should also establish a standard for quality translation services and interpreters. This paper will review current federal regulations and draft policy, analyze literature describing hospital experiences, and discuss non-compliance areas.

(C) 2022 Suzanne Mistretta. This is an open access article distributed under the terms of the Creative Commons Attribution License, which permits unrestricted use, distribution, and reproduction, provided the original author and source are credited. 


\section{Draft Guidance}

In 1995, HHS issued a policy memo clarifying the informed consent process for individuals who do not speak English. ${ }^{4}$ The policy states that the witness required for the signing of consent documents should be fluent in both languages and that the study team should issue a translated short form. ${ }^{5}$ The guidance still does not require an official translator in the short-form process. However, if used, the translator may serve as witness as well. ${ }^{6}$ The policy does not mention the translator's language proficiency requirements or whether the institution must provide the translator. ${ }^{7}$

In 2014, HHS issued draft guidance to expand upon the requirements for informed consent. Per its disclaimer, the guidance is non-binding and not intended for implementation. ${ }^{8}$ First, the guidance attempts to clarify the phrase "language understandable", by adding "the information presented to potential subjects is in a language, and at a level they can comprehend, including an explanation of scientific and medical terms." 9 The draft guidance also notes that all potential research subjects needing translation might have a low level of health literacy and education.

Suppose the research subjects expect a non-English speaking group of participants for enrollment. In that case, the guidance suggests that the researchers provide a long-form translation of study materials before an institutional review board (IRB) initial review of the "appropriately translated consent documents (i.e., either a long form or a short form with written summary)." 10 To satisfy the guidance, researchers would need to provide multiple translations of the same document as the study progresses and the IRB proposes edits to the long-form English document. Because translating the informed consent forms is costly and timeconsuming, having a long form available is not always feasible or practical.

Foreseeing this problem, HHS outlined three steps for what to do when a subject's language is not expected or planned for in the population. First, the guidance suggests that the investigator "determine that there is Sufficient Justification to Enroll the Subject Without Using a Translated Long-form to Document the Subject's Informed Consent." 11 Although this provision protects non-English-speaking subjects from uninformed consent, it adds a barrier to enrollment. The investigator must justify the registration of the individual based on the individual's language. The justification process may delay enrollment, placing individuals at a disadvantage based on their language. If the researcher can adequately justify and use the short-form consent process, the investigator must then translate the long-form consent. After the subject starts participating in research, the investigator then provides the long-form translated document.

\section{Short Form and Noncompliance}

Vagueness in the federal regulations has caused disparate interpretation among institutions, leading to noncompliance. ${ }^{12}$ Each institution has its own interpretation of 45 CFR 46, also known as the Common Rule, which protects vulnerable research subjects and provides research teams with a short-form template. ${ }^{13} \mathrm{As}$ noted above, the regulations do not require a translator, nor do they specify the translator's specific role or qualifications. ${ }^{14} \mathrm{HHS}$ has not made it clear whether the witness may be a member of the study team or related to the patient, or whether the witness providing interpretation must be independent. Furthermore, it is not clear if the witness is overseeing merely the signing of the document or serving as a witness to the informed consent process. ${ }^{15}$ In review of noncompliance areas, the institution should clarify the use of shortform consent, the witness's role, and impose qualification requirements for translators. ${ }^{16}$ Each institution will have its own resources and interpretations. Therefore, non-English speakers will find different translation quality across many research institutions. An individual may receive a different research experience and quality of language resources based on location and the degree to which their language was expected by the research team. This barrier is an implicit form of discrimination and violates the principle of justice. 
III. Equitable Selection of Subjects

Minority populations are underrepresented in research due to a lack of cultural competence and language barriers to subject recruitment. ${ }^{17}$ Paradoxically, minority populations are most impacted by many of the diseases for which there are clinical trials. ${ }^{18}$ In 2015, researchers surveyed 10,000 studies registered on clinicaltrials.gov. ${ }^{19}$ The authors found, "English fluency requirements have been increasing over time, from $1.7 \%$ of trials having such requirements before 2000 to $9.0 \%$ after $2010 .{ }^{\prime 20}$ Researchers who often exclude non-English speaking ethnic minorities claim that diversity may impact whether the studied intervention's effect is noticed. ${ }^{21}$ This manipulation of the subject population goes against the principle of justice outlined in the Belmont Report. Of 14,367 clinical research studies registered on clinicaltrials.gov between 2010 and 2020, 18.98 percent required subjects to be fluent in English. ${ }^{22}$ Regulations and institutions do not compel researchers to include non-English speaking subjects; the researcher is not breaking any federal regulations by excluding them. From an ethical standpoint, the deliberate exclusion based on an inability to read/write in English is inequitable and unjust.

In 2018, researchers reviewed enrollment rates and staff competency for subject enrollment. ${ }^{23}$ Researchers found low levels of cultural competency among research staff and a misunderstanding of the federal regulations. ${ }^{24}$ In 2005, researchers reviewed the importance of cultural competency training for healthcare providers. ${ }^{25}$ Authors synthesized data based on 34 programs and found that competency programs improved provider "knowledge, attitudes, and skills, and patients' ratings of care." ${ }^{26}$ However, cultural competency programs did not improve or impact patient compliance and health outcomes. ${ }^{27}$ Authors requested more research to evaluate cultural training's impact on researcher bias and attitudes toward subject inclusion. ${ }^{28}$

IV. Institutional Barriers

In 2018, researchers conducted a review of approximately 1,500 clinical trials at Boston Children's Hospital and its Anesthesia Research Unit to identify enrollment barriers for non-English-speaking subjects. ${ }^{29}$ The researchers discovered that the number of potential non-English speaking subjects increased, while the number of studies approved to enroll non-English speaking subjects did not grow at the same rate. ${ }^{30}$ Of 1,492 studies that were open to enrollment between 2011 and 2016, 714 did not allow non-English speaking subjects. ${ }^{31}$ The institution cited six barriers to enrollment that precluded these minority-language populations (Table 1). ${ }^{32}$ 
TABLE 1 Definition and distribution of the most common barriers noted by the institution and the Pediatric Anesthesia Research Center studies for inclusion of non-English-speaking patients in research

\begin{tabular}{|c|c|c|c|}
\hline \multicolumn{4}{|c|}{ Distribution of Barriers within Institutional and Pediatric Anesthesia Research Center Studies } \\
\hline Barrier & Definition of the barrier & $\begin{array}{l}\% \text { of studies within } \\
\text { the Institution }(n=714)\end{array}$ & $\begin{array}{l}\text { \% of studies within Pediatric } \\
\text { Anesthesia Research Center }(n=12)\end{array}$ \\
\hline Category 1 & $\begin{array}{l}\text { no funding/resources for translation: financial limitations } \\
\text { or limitations from a study sponsor }\end{array}$ & 9.94 & 0.00 \\
\hline Category 2 & no clear justification provided & 17.79 & 16.67 \\
\hline Category 3 & $\begin{array}{l}\text { study tools/materials are not validated in other } \\
\text { languages: studies indicated that the tools or materials } \\
\text { used as part of the study were not available in other } \\
\text { languages, or translating to other languages would affect } \\
\text { the comprehension of the tool/would confound the results, } \\
\text { or the tool would not be accurate across languages. }\end{array}$ & 35.99 & 58.33 \\
\hline Category 4 & $\begin{array}{l}\text { target population only speaks English: studies indicated } \\
\text { that the population was extremely small and translation } \\
\text { would not make sense }\end{array}$ & 26.89 & 0.00 \\
\hline Category 5 & presumed unavailability of translators & 2.94 & 8.33 \\
\hline Category 6 & $\begin{array}{l}\text { pilot study: studies indicated that there were plans to } \\
\text { translate for larger studies in the future }\end{array}$ & 6.44 & 16.67 \\
\hline
\end{tabular}

Pediatric Anesthesia Research Center studies are also counted within the Institutional Data.

The barriers described in Categories 1, 3, and 5 are due to a lack of translators, funding, or validated translated material. Categories 2, 4, and 6 may reflect an implicit bias on the institution's part and suggest a lack of cultural understanding.

Some researchers ${ }^{33}$ called on the institution to increase cultural awareness and to provide resources to address the effects of the language barrier on enrollment. ${ }^{34}$ However, with no set standard on the institutional level, it is up to the researchers to address this discrepancy and uphold the principle of justice. The uneven distribution of resources requires the research subjects to counteract their own language barrier. In many clinical cases, as time is of the essence, a person may need to seek several research options before participating in their desired study. Until each institution discloses its barriers and noncompliance, we have no way of knowing the full extent of this problem.

\section{Policy Recommendations}

The current HHS regulations and draft guidance are not sufficient to resolve implicit bias and logistical problems that arise when enrolling non-English speaking subjects. HHS should take the following actions. First, HHS should translate short-form consent forms for the most common languages in the US. HHS should then publish the translated documents for use as a template on its website for centers that lack adequate translation resources. Second, HHS should create a federal database of qualified translators who meet language proficiency benchmarks established by HHS. Individuals listed on this database should provide written translation of study materials and verbal translations during the consent process. The database should contain individuals affiliated and unaffiliated with an institution to track credentials and verify language proficiency. Third, HHS should create a federal fund for institutions to draw from when they need resources for long-form translations or translators.

HHS should amend the Common Rule to add specificity. It should define the role of the witness in the shortform consent process to eliminate unequal interpretations across study sites. Second, it should mandate that the IRB obtain strong justification for the exclusion of subjects based on English-fluency criteria. Third, it should recommend cultural competency programs for investigators at the institutional level. 
VI. Limitations

The proposed actions and policy may face objections from the federal government and industry. At the federal level, the government may not have the resources or funding needed to establish a nationwide registry of translators or quality check for fluency. HHS may need to develop a separate office to track these credentials and select a proficiency baseline. Perhaps an independent organization should be contracted by the government to perform these quality checks on a regional basis and call on the states to help fund the project. Also, the federal government may not have adequate resources to create a national fund for institutional-level translation services. One solution may be to include a flat rate of translation in the site budget during start-up activities. The government would be responsible for translations for governmentsponsored research when the institution cannot feasibly provide a translation. Research centers can pull from the translator database for institutional studies if they cannot translate in-house. For pharmaceutical corporation-led protocols, industry sponsors may not appreciate the added cost of translation. However, by mandating the fee, the government would eliminate systemic discrimination based on English fluency.

VII. Support

Researchers should show support for more specific guidance on the institutional level, granted they receive adequate resources to meet the regulations. By clarifying the use of a translator and the role of a witness, and establishing common resources, every institution will benefit. Furthermore, it is crucial to hold institutions accountable for supporting cultural competency initiatives. These programs do not need to be intensive or expensive; there are many resources available to the public on websites such as YouTube. Institutions should make cultural competency programming a mandatory requirement for staff training during the orientation and continuing education. Encouraging cultural competency programs will improve provider attitudes and subject treatment.

\section{CONCLUSION}

Federal regulations do not require the use of resources when enrolling a subject who is non-English speaking, nor do they mandate the inclusion of "unexpected" populations based on language. Lack of clarity and specificity on the federal level places the onus of responsibility on the institution to mitigate language barriers. The lack of universal policy leads to an unequal research experience among institutions and locations. Furthermore, clinical trial sponsors may manipulate data based on limiting the study population and engaging in studies that overly represent homogeneous populations. Influencing the study population will skew results and will not lead to generalizable knowledge. To uphold the principle of justice, the research community must gather its resources to support non-English speaking subjects who wish to participate in research.

\footnotetext{
1 "45 C.F.R. 46 FAQs," 45 C.F.R. §46.116 (a)(3), Office for Human Research Protections, last modified 2018, https://www.hhs.gov/ohrp/regulations-and-policy/guidance/faq/45-cfr46/index.html\#: :text=Basic\%20regulations\%20governing\%20the\%20protection,were\%20first\%20published\%20in\%201974.

2 Office for Human Research Protections, "45 C.F.R. 46 FAQs."

3 "Protection of Human Subjects, 45 C.F.R.§ 46," United States Department of Health and Human Services, last modified 2018, https://www.ecfr.gov/cgi$\mathrm{bin} /$ retrieveECFR $? g p=\& S I D=83 c d 09 e 1 c 0 f 5 c 6937 c d 9 d 7513160 f c 3 f \& p i t d=20180719 \& n=p t 45.1 .46 \& r=P A R T \& t y=H T M L$.
}

${ }^{4}$ Office for Human Research Protections, 45 C.F.R. §46.117(b)(2), “45 C.F.R. 46 FAQs.”

${ }^{5}$ The researcher can orally present the information to the subject or LAR, with a witness present, and provide a short-form of consent with a brief written summary of the research. 
6 "Informed Consent of Subjects Who Do Not Speak English," Office for Human Research Protections, last modified February 25, 2016, https://www.hhs.gov/ohrp/regulations-and-policy/guidance/obtaining-and-documenting-infomed-consent-non-englishspeakers/index.html.

7 Lad, Pramod M., and Rebecca Dahl. "Overcoming Language Barriers in the Informed Consent Process: Regulatory and Compliance Issues With the use of the 'Short Form.'” Accountability in Research 21, no. 5 (2014): 315-320. https://doi.org/10.1080/08989621.2013.848801.

8 "Informed Consent Information Sheet Guidance for IRBs, Clinical Investigators, and Sponsors Draft Guidance," Office of the Commissioner, last modified 2014, https://www.fda.gov/media/88915/download.

${ }^{9}$ Office of the Commissioner, "Informed Consent Information Sheet Guidance."

10 Office of the Commissioner, "Informed Consent Information Sheet Guidance."

11 Office of the Commissioner, "Informed Consent Information Sheet Guidance."

12 Lad and Dahl, "Regulatory and Compliance Issues."

13 Lad and Dahl, "Regulatory and Compliance Issues."

${ }^{14}$ Lad and Dahl, "Regulatory and Compliance Issues."

15 Lad and Dahl, "Regulatory and Compliance Issues."

16 Lad and Dahl, "Regulatory and Compliance Issues."

${ }^{17}$ Staples, Jeanine N. et al., "Language as a Barrier to Cancer Clinical Trial Accrual: Assessing Consenting Team Knowledge and Practices for Cancer Clinical Trial Consent Among Low English Fluency Patients." Applied Cancer Research 38, no. 1 (2018): 1-7. https://doi.org/10.1186/s41241-018-0065-9.

18 Staples et al., "Language as a Barrier."

${ }^{19}$ Egleston, Brian L. et al., "Characteristics of Clinical Trials That Require Participants to be Fluent in English." Clinical Trials 12 , no. 6 (2015): 618-626. https://doi.org/10.1177/1740774515592881.

20 Egleston et al., "Characteristics of Clinical Trials."

${ }^{21}$ Egleston et al., "Characteristics of Clinical Trials."

${ }^{22}$ Muthukumar AV, Morrell W, Bierer BE (2021) Evaluating the frequency of English language requirements in clinical trial eligibility criteria: A systematic analysis using ClinicalTrials.gov. PLoS Med 18(9): e1003758.

https://doi.org/10.1371/journal.pmed.1003758 https://doi.org/10.1080/08989620600654043.

${ }^{23}$ Staples et al., "Language as a Barrier."

${ }^{24}$ Staples et al., "Language as a Barrier."

${ }^{25}$ Beach, Mary Catherine et al., "Cultural Competency: A Systematic Review of Health Care Provider Educational Interventions." Medical Care 43, no. 4 (2005): 356. https://doi.org/10.1097/01.mlr.0000156861.58905.96.

${ }^{26}$ Beach et al., "Cultural Competence," 8.

${ }^{27}$ Beach et al., "Cultural Competence," 8.

${ }^{28}$ Beach et al., "Cultural Competence," 8.

${ }^{29}$ Bernier, Rachel et al., "Inclusion of Non-English-Speaking Patients in Research: A Single Institution Experience." Pediatric Anesthesia 28, no. 5 (2018): 415-420. https://doi.org/10.1111/pan.13363.

30 Bernier et al., "Inclusion of Non-English-Speaking Patients." 6 
Mistretta, Amending Federal Regulations to Counteract language Barriers, Voices in Bioethics, Vol. 8 (2022)

${ }^{31}$ Bernier et al., "Inclusion of Non-English-Speaking Patients."

32 Bernier et al., "Inclusion of Non-English-Speaking Patients."

33 Lad and Dahl, "Regulatory and Compliance Issues."

${ }^{34}$ Bernier et al., "Inclusion of Non-English-Speaking Patients." 